\title{
artículos
}

\section{¿Es necesario un nuevo paradigma en catalogación?}

Ramiro Lafuente

Investigador del CUIB

\section{RESUMEN}

A par tir del análi sis de las rup tu ras y con tra dic ciones con cep tua les ge ne ra das por la in tro duc ción de la com pu ta do ra en el cam po de cata loga ción, el au torrea li za al gu nas propues tas en ca mi na das a re plan tear y re de finir las no cio nes y fi na li da des de la ca ta lo ga ción y normatividad. Se sugiere la posibilidad de la construcción de una teoría sobre catalogación apropiada para dominar la innovación tecnológica que significa la computadora.

\section{ABSTRACT}

From the analysis of con cep tual breaks and con tra dic tions ge ne ra ted by the in tro duc tion of the com pu ter in the field of ca ta lo ging, the au tor ma kes se ve ral pro po sals ai med at res tar ting and re de fi ning no tions and ob jec ti ves in ca ta lo guing. The pos si bi li ty is sugges ted on the building of a theory for cataloguing appropriate to the technological innovation which the computer has meant.

La normatividad para la descripción de materiales bibliotecarios, noes un tema de finitiva men te gla mou ro so... aun que no deja de tener ciertos encantos. Uno de ellos estriba en la posibilidad de replantear las cuestiones sobre < normatividad> en catalogación, para res ca tar las de los es tre chos lí mi tes en el cual se les ubica, y ana li zar las en un ám bi to ma yor co rres pon dien te a las finalidades a cumplir por la catalogación.

Al examinar el concepto de normatividad en catalogación, se toma como pun to de par ti da las rup tu ras y con tra dic cio nes concep tua les ge ne ra das por la in tro duc ción de la com pu ta do ra en el campo de la catalogación.

Se con si de ra la au to ma ti za ción como una in no va ción tec noló gica cuya in fluen cia va más allá del uso de una téc ni ca para el proce so de da tos, pues se es ti ma que ge ne ra un con jun to de es truc turas lógicas para procesar y ordenar los elementos de la descripción y clasificación documental, y procedimientos para la organización del trabajo de catalogación que significan cambios en las con cep cio nes bá si cas acer ca de los pro ce sos para describir y clasificar documentos.

Eluso cre cien te de me dios com pu ta ri za dos para re sol ver los proble mas de riva dos delcon trolbiblio gráfico, orien ta dos afa cilitar el in ter cam bio de in for ma ción de ri va da de la des crip ción y cla sificación de documentos; plantea la necesidad de nuevos esque- mas conceptuales para solucionar aquellas cuestiones propias del uso de mediosautomatizados, que rebasan los planteamientos previstos en el control bibliográfico por medios manuales.

Lain tro duc ción de má qui nas com pu ta do ras para apo yar trabajos de catalogación, suscitó múltiples reflexiones y controversias, de ri va das de las ade cua cio nes que fue ne ce sa rio efec tuar a las reglas para catalogar, con objeto de facilitar el uso de la máquina. Sin embargo, fue la adopción cotidiana de medios automatizados como par te de las ta reas de ca ta lo ga ción, lo que in flu yó en la creación de nuevos conceptos y provocó modificaciones a los procesos de catalogación.

Empero como la automatización de procesos de catalogación, lle gó a un pun to, en el cual no re sul ta ade cua do con ti nuar por el ca mi no de ajus tar las re glas de ca ta lo ga ción con ob je to de fa ci litar el uso de la má qui na, sur gen cues tio na mien tos en ca mi na dos a replantear definitivamentelas nociones y finalidades de la catalo ga ción, con la in ten ción de cons truir una teo ría so bre ca ta lo gación apro pia da para do mi nar la in no va ción tec no ló gi ca que significa la computadora. 


\section{ORIGEN Y DESENLACE DE UN PARADIGMA}

Tradicionalmentelacatalogación des criptivaseconsideracomo un quehacer estrictamente técnico circunscrito al ámbito de la apli ca ción in fle xible de nor mas y re glas para ca ta lo gar... las cuales constituyen un conjunto de bien definidos procedimientos para construir catálogos.

Una cuidadosa revisión de la historia de la catalogación nos mues tra que el ori gen y de sa rro llo de las re glas para ca ta lo gar es un tema con tro ver tible, sin em bar go los obje ti vos pro pues tos por <Cutter y los Principios de París>, podríamos considerar que cons ti tu yen un para dig ma so bre ca ta lo ga ción por que tie nen a su favor su aceptación y uso más o menos generalizado.

La idea de una es truc tu ra li neal del ca tá lo go, sus ten ta da en elautor-título como ele men to cla ve para la or ga ni za ción y bús que da en un catálogo, se mantiene hasta la fecha. Diferentes códigos para catalogar, como las AACR1, AACR2, el código alemán RAK, son si mi la res en este as pec to cla ve (Da vies (1987), p. 89),

Estimamos que Cutter logra a principio del siglo XX sistematizar el saber técnico para hacer catálogos por medio de tarjetas, que sus ti tu yen a los ca tálo gos en for ma de libro, y de fi ne con ceptos acerca del significado de la catalogación.

Para Cutter "catalogar es un arte, no una ciencia. Ningunaregla toma el lu gar de la ex pe rien cia y el jui cio, pero al gu nos de los resultados de la experiencia pueden ser recomendados por las reglas" (Cutter, p. 6).

En la de fi ni ción de Cut ter so bre el acto de ca ta lo gar pre va le ce la idea de englobar soluciones de saber técnico, construidas por medio del agrupamiento de reglas, producto del saber empírico acu mu la das a tra vés del tiem po, a las cua les les atri bu ye una va lidez "uni ver sal", de ri va da de la efi ca cia de mos tra da por la ex perien cia. Al con si de rar la ca ta lo ga ción como un arte re co no cía las limitaciones de los conceptos para organizar catálo gos de tarjetas, y su je ta ba a la "ex perien cia y buen jui cio" del ca ta lo ga dor las posibilidades para resolver las cuestiones no previstas.

Exis te im plíci ta en las con cep cio nes de Cut ter la idea de la im posibilidad de subs ti tuir la ex perien cia para ca ta logar porcual quier cuer po teó ri co con cep tual, que hi cie ra fac tible tan to la com prensión del problema como su explicación y enseñanza. Prevalece en Cutter la idea arraigada en los bibliotecarios del siglo XIX acer ca de la eru di ción como un fac tor im por tan te para el lo gro de una eficaz organización bibliotecaria.

Estacon cepción de la ca ta log a ción comouna ac ti vidadempírica su je ta a re glas para la solu ción de ca sos pre via men te ana li za dos; no fue la úni ca, en esa mis ma épo ca el ale mán Ru dolf Focke había pro pues to otro tipo de res pues tas ba sa das en la idea del ca tálo gocla sifica do, y fue el pri me ro en po nerén fa sis en las po si bilidades de la teoría como medio para descubrir y corregir la inconsistencia de los catálogos; sin embargo la Primera Guerra Mundial impidió la consolidación de sus conceptos y favoreció el afianzamiento de las propuestas recogidas por Cutter (véase Stevenson).

La construcción de catálogos agrupando reglas representativas de un saber técnico compuesto de procedimientos que convierten la catalogación en una actividad reducida exclusivamente a la apli ca ción de la re gla, no sólo in flu yó en la or ga ni za ción de los catálogos; con el transcurso del tiempo absorbió lentamente las concepciones acerca de las finalidades sociales de la biblioteca, has ta lo grar que la no ción me ra men te téc ni ca de la bi blio te ca se convirtiera en una idea dominante.

Al abor dar los pro ble mas de la or ga ni za ción bi blio te ca ria como cues tio nes cuya so lu ción se en cuen tra en la apli ca ción de un procedimientotécnico y abandonar la idea de la eru di ción como un componente inherente a la tarea del catalogador, se generó al mis mo tiem po una di vi sión del tra ba jo que co rres pon die se a esa <naturaleza técnica de la biblioteca>. Un pri mer paso lo cons tituyó el se pararcon fi nes de con trol ad mi nis tra ti vo, las ac ti vi da des de catalogación, administración y servicios.

Si bien esta división del trabajo permitió aumentar la <eficiencia> de la organización bibliotecaria, sin embargodeteriorónotablemente la actuación social y formación profesional del bibliotecario, y fragmentó el desarrollo del conocimiento bibliotecológico en estancos independientes unos de los otros con escasas posibilidades de interrelacionarse; centrando la inves ti ga ción y en se ñan za de la bi blio te co lo gía en as pec tos me ra menteoperativos, relativosalfuncionamien todelasbibliotecas.

No es ob vio el ca mi no que se tran si tó has ta con fi gu rar una si tuación de tal naturaleza, es aún un tema que requiere una amplia explicación por parte de la historia de la catalogación, porque sig ni fi có la acep ta ción por la fuer za de la cos tum bre y la pre sión so cial de mu chos su pues tos acer ca del tra ba jo del biblio te ca rio y de su si tua ción como em plea do pú bli co o aca dé mi co y de sus finali da des so cia les como profe sional, to dos ellos im plíci tos en la construcción y desarrollo de las reglas para integrar catálogos, como instrumentos de organización bibliotecaria.

Uno de los aspectos esenciales que caracterizan la constitución de una bi blio te ca, es triba en la or ga ni za ción de sus colec ciones... esta or ga ni za ción se so por ta fun da men tal men te en los prin ci pios derivados de los conceptos sobre catalogación. Son por tanto elementos determinan tes paralacon formación deconcepciones sobre la biblioteca.

La catalogación ha sido el sustentoindispensable para instaurar los ser vi cios de la bi blio te ca. El ac ce so a las co lec cio nes de pen de delca tálogo, e in clu si ve mu chos de los con troles ad mi nis tra ti vos se de ri van deéste (el ca tálogo de ad qui si ciones, el ca tálo go to pográ fi co para in ven ta rios, por ej.). Una de las pri me ras y más impor tan tes rup tu ras se pro du jo con la in tro duc ción de la idea de la es tan te ría abier ta, en don de la cla si fi ca ción ad quie re un pa pel rele van te para or ga ni zar y per mi tir el ac ce so a las co lec cio nes de la biblioteca, independientemente del catálogo. 
La catalogación como instrumento para la organización de las colec ciones de unabiblio teca, se en cuen train se parable menteli ga da a las fi na li da des de la bi blio te ca, por que ésta siem pre se ordena para cumplimentar determinado tipo de fines.

El pro pó si to esen cial de la ca ta lo ga ción es pro por cio nar al pú blico los medios para consultar las colecciones de una biblioteca, sin em bar go las re glas para des cri bir docu men tos y or ga ni zarcatá lo gos par ten de ideas rí gi das acer ca de las for mas de bús que da de do cu men tos por par te del usua rio, sin abun dar en los pro blemas inherentes a cómo satisfacer las demandas de información generadas por el público que hace uso de la biblioteca.

Los conceptos para la descripción y clasificación de los documentos así como para el ordenamiento de catálogos, se fundamenta en ideas sumamente rígidas acerca de las formas de búsqueda de información por parte del público. De esta manera las re glas para ca ta lo gar con tri bu yen a con fi gu rar una idea de usuario, implíci ta en las re glas, peroin su fi cien te men te de fi ni da, con lo cual los servicios bibliotecarios se orien tan a un usua rio cuya de finición úni ca men te pue de des pren derse de los su pues tos contenidos en las reglas. Lo cual contribuye a conformar una paradoja acerca del usuario y los servicios bibliotecarios.

Las estructuras de los catálogos se fundamentaron en la idea de un orden in fle xi ble para el re gis tro de los da tos, este or den es el sustento de la organización del catálogo, por tanto cualquier trans gre sión al mis mo, afec ta su or ga ni za ción y mo di fi ca su capacidad de recuperación.

Como las estructuras utilizadas para la organización de catálogos carecen de flexibilidad para aceptar modificaciones no previs tas, la re gla debe apli car se li te ral men te, por que de no ha cer lo se afecta el poder de recuperación del catálogo, cualquier cambio no con tem pla do hace que la in for ma ción se pier da en la or ganización del catálogo.

Este problema lo advirtió Cutter cuando mencionaba que "La con ve nien cia del pú bli co debe es tar por en ci ma de la del ca ta loga dor. En mu chos ca sos coin ci den. Unas re glas sen ci llas sin excepciones no sólo son fáciles de trabajar, sino fáciles de entender. Pero la es tric ta con sis ten cia de una re gla y la uni for mi dad en su aplicación algunas veces conlleva prácticas que chocan con las prácticas habituales que tiene el público de buscar las cosas. Cuando estos hábitos están su ma men te en rai za dos, es ton to por parte del catalogador ignorarlos y demandan de él un sacrificio del sistema y su simplicidad" (Cutter, p. 6).

De lo dicho por Cutter se desprende que la comprensión de los hábitos de búsqueda por parte del público, debe ser algo excepcional, y acep tar los cuan doel ca ta lo ga dor no ten ga otro re me dio, aun cuan do ten ga que sa cri fi car la apli ca ción exac ta de un sis tema para hacer catálogos, que sería su objetivo primario.

Al su je tar la cons truc ción de ca tálo gos al cum pli mien to es tric to de reglas, dejando a un lado la posibilidad de conservar una visión in te gra do ra que per mi tie ra en glo bar los fe nó me nos y fi nalida des del queha cer del ca ta loga dor, con los ob je ti vos de servi cio de la biblioteca, se fomentó la implementación de soluciones téc ni cas des vin cu la das de apre cia cio nes teó ri cas que pu die se articular la actividad del catalogador con lasfinalidades a cumplir por la biblioteca.

La separación del trabajo del catalogador de cualquier intento porcom pren der los hábi tos de bús que da del público, es pecializó su tra bajo y elúni co con tac to que man tie ne con las fina li da des de la bi blio te ca se da a tra vés del ca tálo go, in clu si ve las ne ce si da des del usuario están preconformadas por medio de las reglas para elaborar el catálogo.

Como el esfuerzo realizado por el catalogador comenzó a tener un rendimiento decreciente para efectos de recuperación de información documental, fueron surgiendo actividades que crearon estancos aislados, que antes se encontraban como parte de los proce sos de ca ta lo ga ción, tal es el caso de los ín di ces. In clu so se fragmentó la idea de la biblitoeca y propició el desarrollo de proyectos como los centros de documentación.

La mayor par te de las so lu cio nes en cues tio nes de ca ta lo ga ción, se en cuen tran re fe ri das al proble ma en símis mo, sin ocu par se de sus oríge nes o fi na li da des. Como se par te de re glas re la cio na das ex clu si va men te con la for ma de or de nar una ca de na de da tos que describe un documento, la descripción documental se convierte en una ac ti vi dad ca suís ti ca de loca li za ción de la re gla, sin nin gut na otra posibilidad.

De esta ma ne ra la ca ta lo ga ción se en cie rra en un cír cu lo vi cio so, porque la elaboración de un ca tá lo go, no tie ne otra sa li da que la apli ca ción de la re gla. Cual quier in ten ción de cues tio nar la re gla o desarrollar un estudio teórico de la misma, resulta un intento fútil, porque en última instancia cuestionar cualquier regla en for ma ais la da ori lla el análi sis a un tra ta mien to ca suís ti co in finito.

Al con ver tir se la prác ti ca de la des crip ción do cu men tal y la construc ción de ca tá lo gos en un fin en sí mis mo, se re fuer za la cre encia de que las formas de búsqueda de información implícita en las reglas de catalogación, corresponde a necesidades reales.

El pro ce so para bus car in for ma ción no se pue den re du cir a los estre chos es que mas que es fac ti ble vis lum brar a par tir de la téc nica para catalogar, porque las formas de indagar para encontrar un dato, es un problema ins cri to en cues tio nes mu cho más am plias con cer nien tes por un lado a las for mas en cómo y por qué se uti liza la información, y algo de lo cual realmente sabemos muy poco: <los mecanismos utilizados por el ser humano para indagar acer ca del tipo de da tos o in for ma ción que le in te re sa> (véa se Brooks).

El análisis del agrupamiento de las reglas catalográficas no es una cuestión libre de escollos, porque muchos de los conocimientos técnicos provienen de la práctica cotidiana que con el trans cur so del tiem po y de bi do a la com probación em pírica de su funcionalidad, se convierten en reglas al adoptarse para su uso por varias entidades. 
Mu chas re glas para cons truir ca tálogos tien den a ser co difica ciones de las mejores prácticas conocidas. Sin embargo la influencia de la cien cia en ge ne ral, y de los co no ci mien tos so bre la administración de las bibliotecas en particular, influye en la creación de re glas, por lo que es ne ce sario dis tin guiren tre és tas y las que son expresión de las mejores prácticas conocidas en un tiempo (véase Harvard, p. 174).

Em pe ro el aná li sis de las re glas es una cues tión su je ta a di ver sos gra dos de di fi cul tad, de ri va dos de la fal ta de es truc tu ra ló gi ca de las re glas. Los es tu dios rea li za dos para de mos trar que exis te una estructura lógica en las reglas para catalogar, han terminado en in ten tos falli dos que aca ban poreviden ciarcómolaca ta lo ga ción se rea li za con base en agru pa mien tos de re glas, cuya úni ca in tención es con fi gu rar un pro ce di mien to para ha cerca tálogos (véa se Shaw y Davies (1984)).

\section{LOS CUESTIONAMIENTOS A LAS CONCEPCIONES SOBRE CATALOGACION}

Según Roy Davies, tres factores contribuyen a romper el paradig ma exis ten te en el área de ca ta lo ga ción: (1) los avan ces tec noló gi cos, (2) las as cen den tes ex pec ta ti vas de los usuarios, (3) factores de índole económico (Davies (1987) p. 90).

Para Davies, con el catálogo de tarjetas el número de puntos de acceso está restringido, y únicamente es posible una forma de búsqueda. Las nuevas tecnologías, dice Davies, nos liberan de esta herencia; la búsqueda ya no es completamentedependiente de los encabezamientos escogidos por los catalogadores. Además la re le van cia de los có di gos de ca ta lo ga ción exis ten tes se redu ce, aun que si que re mos con tar con lla ves de al ma ce na mien to y re cu pe ra ción se gu ras y efi ca ces, siem pre será ne ce sa rio proceder con for me a cier tas re glas. No obs tan te, la vie ja dis tin ción entre descripción y punto de acceso perdió su fuerza.

Los conceptos mencionados en el párrafo anterior conducen a Da vies a con cluir que la ca ta lo ga ción ne ce si ta po ner se al día con las nue vas prác ti cas de al ma ce na mien to y re cu pe ra ción de in formación, y de sa rro llar prin ci pios teó ri cos efi ca ces para el apro vechamien to de las posibilida desque ofrece el proceso au to matizado (Davies (1987), p. 90).

Michael Gorman había planteado en 1977, que la computadora abría nue vas po si bi li da des, y por tan to nue vos pro ble mas para la biblioteca, al re de fi nir las re glas del jue go, y aun que no ne ce saria men te lo hace mas fá cil de ju gar, ideal men te con vier te el juego en más prove cho so para el par ti ci pan te y el es pecta dor (Gorman, p. 588).

Para Gor man las nue vas tec no lo gías y cir cuns tan cias so cia les requieren un redefiniciónde nuestros catálogos, y es esencial que el nue vo plan sea di se ña do den tro de la gran tra di ción de Pa nizzi, Cutter y Lubetzky (Girnabm o, 601).

Según Vickery, un registro de máquina no es simplemente una nueva forma de almacenar y manejar los tradicionales registros de asiento bibliográfico, es un medio físico nuevo y diferente, que tie ne usos dis tin tos a los re gis tros tra di cio na les. La úni ca razón para crear registros automatizados, es que la máquina nos ofrece la posibilidad de usar los registros bibliográficos de maneras distintas (Vickery, p. 428).

El re gis tro au to ma ti za do nos abre una ma yor, más hol ga da y flexible mani pulación de los datos. Debido a estafle xibilidad, an tes de decidir un formato automatizado, debemos especificar los pro pó si tos a los que pue den ser vir los re gis tros bi blio gráfi cos, y las formas que pueden o deben tener para servir a estos propósitos (Vickery, p. 428).

Para Vickery un re gis tro ca ta lográ fi co tra di cio nal es una en tra da de catálogo, es algunas veces casi una descripción narrativa del do cu men to. Los prin ci pios del di se ño para las en tra das del ca tálo go, han sido de sa rrolla das para cada fun ción ais la da men te, sin embargo para poder diseñar un óptimo registro bibliográfico es ne ce sa rio con si de rar to das las fun cio nes del ca tá lo go y sus po sibles interacciones en forma simultánea (Vickery, p. 428).

En opi nión de Vickery, la po si bi li dad de la ma ni pu la ción de registros bibliográficos por medios automatizados, requiere reconsiderar las estructuras del catálogo, porque para convertirlo en un for ma to de má qui na, ine vi table men te debe mo di fi car se su estructura. Has ta aho ra, las mo di fi ca cio nes han ido en di rec ción de man te ner el for ma to tra di cio nal del ca tá lo go aña dién do le lla ves ex tras para la re cu pe ra ción de da tos, y mo difica cio nes orienta das a faci li tarel or de na mien to de da tos por me dios au to ma tizados (Vickery, p. 429).

Este cam bio de apre cia cio nes en cuan to a los pro ble mas de ca talogación, se origina al perder el catálogo su identidad separada. Mien tras el ca tálogo de tar je tas es un obje to con cre to, su au to mati za ción lo con vier te en un in tan gi ble, una he rra mien ta para facili tar de ter mina das ope ra ciones o servicios, que lo des pojan de su característica de objeto único con finalidades específicas.

La automatización contribuyó a modificar las apreciaciones sobre el catálogo porque la complejidad de los sistemas de información y comunicación, magnifican la ineficiencia en la representación de los datos. La ambigüedad en el uso de los códigos de representación de datos de la descripción documental es una fuente de estas ineficiencias (Tannehill, p. 285).

Si un ele men to de des crip ción es uti li za do como lla ve de re cu peración por medios automatizados, su representación debe ser con sis ten te para que sea po si ble lo ca li zar to dos los ca sos de ocurrencia de un elemento dentro de un archivo. Esto requiere que las lla ves de in for ma ción sean sim bó li cas de tal ma ne ra que puedan ser arre gla das en un or den con ven cio nal, y cuan do un mis mo elemento se presente en más de un re gis tro, éste se en cuen tre representado por símbolos idénticos o que su presencia pueda ser ligada.

Es tos re que ri mien tos cor tan el tra di cio nalén fa sis so bre la transcripción de los datos exactamente como aparecen en el docu- 
mento. Si la normalización es esencial para la recuperación, la demanda por una exacta transcripción puede dejarse de lado (véase Vickery, p. 432).

Lacre cien te pre sen cia de ca tálo gos au to ma ti za dos obliga a re visar los conceptos de catalogación ac tua les, pero para asi mi lar la innovacióntecnológicaque representalaautomatización, resulta inevitablereconsiderar el proceso de catalogación, y contemplarlo como una actividad en donde se conjuntan tanto las formas como las funciones y finalidades del catálogo. El catálogo ya no se ría un ente ais la do cu yas fi na li da des y prin ci pios se agoten en sí mismo.

Un catálogoautomatizado pue de hacer de sa parecer lain de le ble línea que ha se para dola des crip ción de ma te ria les docu men tales con fines ca ta lo gráficos, de la des crip ción con fines bi blio gráfi cos. Las posiblidades delcatálogo automatizadolotransforman en un conjunto de funciones para atender a la realización de diversos fines a partir de un mismo tipo de datos. Empero si bien di lu ye las diferen cias téc ni cas de la des crip ción y re pre sen ta ción de documentos, ahonda las diferencias entre catalogación y bibliografía, si atendemos a las finalidades de cada una.

Al en glo bar la teo ría so bre ca ta lo ga ción, los pro ble mas de re presentación y organización documental, y liberar a la bibliografía de es tos proble mas, le proporcio na una sus ten ta ción téc nica, que ineludiblemente induce a replantear los problemas de la bibliografía, por que al es tar re suel tas las cues tio nes re la ti vas a los procedimientostécnicos para su elaboración, porme dios au to matizados, adquiere una relevancia mayor al cumplimiento de sus fi na li da des de se lec ción, aná li sis críti co y pre sen ta ción de pa noramas bibliográficos.

Inclusive la creciente presencia social de medios de control biblio gráfi co, re du cen la ne ce si dad de la bi blio grafía, pero pre sionan en el sen ti do de una la bor bi blio grá fi ca orien ta da a dis cri minar información, lo cual involucra a la bibliografía en la ela bo ra ción de ver da de ras sín te sis críti cas de los pro ble mas re levantes abordados en la literatura motivo de recopilaciónbibliográfica, lo cual implica conceder mayor importancia a la represen ta ción de las po si bles re la cio nes en tre los di fe ren tes tex tos y sus contenidos, que a la simple descripción y organización bibliográfica con fines de almacenamiento-recuperación.

La automatización del catálogo induce la creación de nuevas ideas en el ám bi to bi blio grá fi co, como la bi blio me tría y el aná lisis de ci tas, que aun cuan do pue dan ser cues tio na bles en cuan to a su sus ten ta ción teó ri ca y fi na li da des, mues tran un cam bio en las posibilidades de la bibliografía, que indican necesariamente la pre sen cia de nue vas fron te ras y te rre nos inex plora dos en el campo bibliográfico.

El ca tálogo au to ma ti za do que da su je to en ton ces a nue vas re glas do mina das por me tas y finali da des que tras cien den la na tu ra le za del catálogocon si de ra doúni ca men te como un me dio para or ga nizar la in for ma ción con te ni da en unabiblio teca. Sus po sibilidades de almacenamiento, recuperacióny ordenamiento de datos, aumentan y se diversificanconforme a las finalidadesperseguidas.

\section{LAS POSIBILIDADES DE LA TEORIA SOBRE CATA- LOGACION}

Si entendemos la catalogación como el proceso intelectual por me dio del cual un do cu men to se des cribe, ca te go ri za por tema, y se le asig na una lo ca li za ción fí si ca en la bi blio te ca (véa se Fre eman, p. 704). En ton ces la teo ría so bre ca ta lo ga ción ten dría como obje ti vo pro por cio nar nos una me jor com pren sión de este proceso, cuyo resultado fi nal es la ela bo ra ción de un ca tá lo go y la organización de colecciones de materiales bibliotecarios.

La construcción de una teoría sobre ca taloga ción pue de pro porcio nar nos una me jor com pren sión de las fun cio nes y fi na li da des del ca tá lo go, pero por sí mis ma no pue de sus ti tuir el sa ber téc nico, acerca de los proce di mien tos parahacerca tálogos, con te ni do en diferentes códigos y normas.

Uno de los proble mas para la re de fi ni ción de los con cep tos so bre catalogación radica en crear un solo cuer po con cep tual ca paz de englobar las técnicas de descripción documental, así como las es truc tu ras y con cep tos de cla si fi ca ción, a par tir del cual sea factible ori ginarins tru men tos útiles, produc to res de in for ma ción al servicio de la interrogación del lector.

Cualquier intento de construcción teórica de principios de catalo ga ción, im pli ca un cam bio en las for mas de ra zo na mien to para abordar al almacenamiento-recuperación de información documen tal, so bre todo por que será in dis pen sa ble in cluir en es tos replanteamientos la similación de las innovaciones tecnológicas introducidas por la automatización, con objeto de subordinar la tecnología a las necesidades propias de la catalogación y evitar que el proceso de catalogación se vea dominado por la tecnología de automatización.

Una bue na teo ría ca ta lo gráfica, una vez de sa rrolla da, y con frontada con los hechos, para rehacer aquellos conceptos inadecuados, ya sea por que in duz can una fal sa com pren sión de las fun ciones y finalidades de la catalogación o porque conduzcan a prácticasconfusas o con tra dic to rias en la ela bo ración de ca tálogos; serviría como instrumento para valorar la eficacia de un cuerpo normativo para el cumplimiento de fines predeterminados.

El de sa rro llo de la teo ría so bre ca ta lo ga ción con du ci ría a un ordenamiento de conceptos útiles para estimar cuándo un cuerpo nor ma ti vo o par te del mis mo pue de o no ser pro ve cho so para el cumplimien to de objetivos decatalogación pre determinados. De esta ma ne ra se con ta ría con ele men tos parajuz gar de ma ne ra ópti ma la vir tud del sa ber téc ni co para ha cer ca tá lo gos, a la vez que se con ta ría con he rra mien tas con cep tua les para orien tar la mo difi ca ción o crea ción de nue vas téc ni cas de or ga ni za ción de ca tálogos, cuando las necesidades prácticas lo hagan necesario.

La teoría sobre catalogación constituiría entonces un principio aglu ti na dor de las téc ni cas para or ga ni zarca tálo gos, a la vez que 
ac tua ría como me dio para ar ti cu lar las con cep cio nes so bre ca talogación a los supuestos y finalidades de la organización y búsqueda de documentos, con lo cual se facilitaría la integración práctica de la catalogación con to dos los ser vi cios que cons ti tuyen la estructura de la biblioteca.

Cuan do se pien sa en la ca ta lo ga ción úni ca men te como una ac tividad encaminada a la descripción y organización de colecciones, como par te de un sim ple for ma lis mo, ais la do o al mar gen de cualquierconsideración sobre la estructura que se está creando, sin ocuparse de las condiciones socia les que facilitan oimpiden su uti li za ción, se está con tribu yen do a for ma li zar es truc tu ras documentales, caren tes de sen ti do, por que las colec ciones, por bien estructuradas que estén, por sí mismas, aisladas de su contexto social, no tienen significación de ningún tipo.

Elcues tiona mien to de los plan tea mien tos señala dos en los párra fos an te rio res, qui zás de bie ran ser los pa sos ini cia les en la de termi na ción de ob je ti vos para el de sa rrollo de una teo ría so bre ca talogación.

Pero el en fo que fun da men tal de las cons truc ciones teó ri cas en el ám bi to de la ca ta lo ga ción no se pue den re du cir úni ca men te a valorar el saber técnico ya establecido, sino primordialmente a orien tar la ac ción para la crea ción de nue vas con cep cio nes so bre los problemasinvolucradosenelalmacenamiento-recuperación de información documental, a partir de las cuales sea posible plantear diversas opciones para la organización de catálogos, que no sean simplemente una versión funcional de lo que ya se está haciendo.

La creación de nue vas op ciones en ca ta lo ga ción es un im pe rativo de ri va do de la di fi cul tad de sa tis fa cer las de man das de in forma ción ge ne ra das por el pú bli co por me dio de las téc ni cas ac tuales para la cons truc ción de ca tá lo gos, por que los he chos re la ti vos al al ma ce na mien to y circu la ción do cu men tal, se han des plaza do delám bi to es pe cífi co del uso de es que mas de cla si fi ca ción ha cia la cap ta ción y re pre sen ta ción del con te ni do del do cu men to, con base en su representación por medio de códigos descriptivos (véase Scarpit, p. 206-207).

Una teoría sobre catalogación únicamente puede ser de utilidad en la medida en que sus concepciones respondan a una política de circu la ción do cu men tal, que tome en con si de ra ción la na tu raleza de la biblitoeca como formadora de colecciones, inabarcables en su con jun to por un solo in di vi duo. Y como con tra par ti da la idea de que un individuo tiene un conjunto de conocimientos registrados en su memoria, los cuales formalmente se encuentran "desordenados", o guar dan un or den que di fi cil men te coincide con el "orden formal" preestablecido por las estructuras para organizar documentos.

Porque como indica Schutz, "la principal característica de la vida de un hombre en el mundo moderno es su convicción de que, en conjun to, su mun do vi tal no es to tal men te com pren sible para él ni para ninguno de sus semejantes. Existe un acervo de conocimiento teóricamente disponible para todos, acumulado por la ex pe rien cia prác ti ca, la cien cia y la tec no lo gía como con- cepciones fundamentales. Pero este acervo de conocimiento no está integrado; consiste en una mera yuxtaposición de sistemas de conocimiento más o menos coherentes, que por su parte no son coherentes, ni siquiera compatibles unos con otros."

El lo gro de la li bre circu la ción do cu men tal, ad quie re una im portan cia sin gu lar para la cons truc ción de una teo ría so bre ca ta lo gación, por que el or den im pues to por el ca tá lo go debe ser ma ne jable por el individuo, al margen de cualquier tecnicismo, que restrinja el li bre ma ne jo de la re cu pe ra ción por par te de los in dividuos; de otra forma se destruye la esencia misma del servicio biblio te ca rio... elfacilitar el ac ce so del público a las colec ciones documentales.

\section{¿QUE SUCEDE CON LA NORMATIVIDAD EN CATA- LOGACION?}

La normatividad en el ámbito de la catalogación constituye un anun cia do que debe for mar par te del cuer po con cep tual de la teoría so bre ca ta lo ga ción, cuyo con te ni do es ta ría orien ta do a la forma ción de con cep tos a par tir de los cua les pue dan de ri var se ac tividades formales de carácter unificador de técnicas (normas) y conceptos (finalidades), así como fomentar las actividades de normalización informal (docencia, investigación, terminología, acuerdos institucionales).

Las normas son instrumentos esenciales para la normalización, pero como re pre sen tan res pues tas con cre tas a proble mas es pecí ficos; es menester establecer generalizaciones que definan los principios con base en los cuales se habrán de articu lar di versas téc ni cas de riva das de dis tin tas nor mas, y po der for mu lar un todo coherente. Las nor mas de ben cum plir la mis ma fun ción de puntos de referencia que cumplen los diccionarios de una lengua.

Los com po nen tes de la nor ma ti vi dad en ca ta lo ga ción de ben susten tar se en la cons truc ción de prin ci pios ge ne ra les, cuyo ob je tivo primordial sería hacer hincapié en la acción unificadora de conceptos, por en ci ma de cualquiercon cep ción téc nica es pecífi ca. No sería ajena tampoco al enunciado de juicios cualitativos que sir van para eva luar la for ma y ob je ti vos a cum plir por un sistema de control bibliográfico, y abarcar los diferentes aspectos de la amplia gama de fines e ntereses a cubrir por una organización do cu men tal, por que la nor mali za ción única men te ad quie re sen ti do cuan do se le vin cu la el uso y des ti no de los do cu men tos.

Aunque estamosacostumbrados a evaluar las actividades de infor ma ción so bre la base de ele men tos cuan ti ta ti vos de los ser vicios prestados, es indispensable encontrar los medios adecuados, de carácter cualitativo que junto con elementos cuantitativos nos ayuden a de termi narsi un sis te ma de in for mación es el indicado para almacenar y usar la información documental disponible (véase: Zweinzing).

La con fi gu ra ción de con cep tos que sir van como me dios para decidir acerca del uso de determinadas normas, no se reduce a un problema de enumeración de criterios de selección acerca de la aplicabilidad monolítica de una norma en detrimento de otra, pues abar ca una gama más am plia de pro ble mas acer ca de la de- 
fi ni ción de cri te rios y li nea mien tos para el uso de téc ni cas y procedimientos de almacenamiento y recuperación.

To me mos, por ejem plo, un he cho: en la so cie dad del si glo XX se ge ne ra ron cam bios en la es truc tu ra de co mu ni cación de in for mación, el aumentarse las posibilidades de producción y circulación de la mis ma, a cos tos re la ti va men te más ba jos que en si glos anteriores.

Este au men to en las can ti da des de in for ma ción dis po ni ble arrastró con si go la idea de con si de rar como un fac tor im por tan te en la transmisión de información, la pre sen cia de una ma yor o me nor cantidad de información. Como consecuencia en el campo del control bibliográfico o documental, se llegó a es ti mar que ne cesariamente la información reduce la incertidumbre.

Esta ma ne ra de pen sar se ve re for za da so bre todo por que los sistemas de control bibliográfico o documental hacen demasiado énfasis en la organización de la información y su provisión al usuario, ex clu yen do cual quier con si de ra ción res pec to a los fe nómenos de comunicación presentes en el proceso de almacenamiento y recuperación de información.

El pres tar una ma yor aten ción a los he chos que go bier nan la produc ción, provi sión y recep ción de in for ma ción docu mental,consi de rán do los como par te de un proce so de co mu nica ción orien tado a satisfacer la necesidad de conocer determinados documentos, nos permi te si tuar un sis te ma de al macenamien to y recu pe ra ción do cu men tal en el cen tro de un am bien te in for ma tivo determinado, y por tanto aumentar las posibilidades de que sea útil para fa mi lia ri zar se con lo que su ce de en dis tin tos cam pos delconocimien tore laciona dos con elcum plimien to de objetivos específicos.

En este sen ti do la nor ma ti vi dad de la ac ti vi dad de cla si fi ca ción y descripción documental debe proveer de instrumentos para deter mi nar y eva luar la efi cien cia téc ni ca de un sis te ma de con trol bi blio gráfi co o do cu men tal, en ra zón de las res pues tas que pue da pro por cio nar al pú bli co, y di ri gir la eva lua ción ha cia sus efec tos favorables (rentabilidad, productividad, etc.) "más en razón de las pér di das que evi tan que de las ga nan cias o pro ve cho ob te nido. Por ejem plo, si un pa sa je ro tie ne una ta bla de ho ra rios y ru tas de tre nes y avio nes, eso no hará que el sis te ma de trans por te vaya más rá pi do, pero pue de evi tar es pe ras in ne ce sa rias. La in for mación so bre ru tas y tiem pos pue de ayu dar a fa ci li tar el via je" (Mikailov, p. 15).

Los servi cios de in for ma ción do cu men tal, me di dos por el grado de pre ven ción de pér di das, ayu da a con fi gu rar un es que ma normativo del control bibliográfico o documental cuya intención principal sea prevenir pérdidas innecesarias.

Me dir los efec tos de un sis te ma de in for ma ción do cu men tal, por me dio de las pér di das que evi ta cuan do la co mu ni dad a la que sir- ve se en cuen tra bien in for ma da y no sólo con base en la can ti dad de in for ma ción que es ca paz de cap tar y or ga ni zar el sis te ma, significa el crear esquemas normativos de evaluación cualitativa.

Generalmente los sistemas de información documental responden a criterios normativos en donde la evaluación de sus actividades se rea li za so bre la base de ele men tos cuan ti ta ti vos del núme ro de ser vi cios pres ta dos. Si la nor ma ti vi dad pre ten de in cluir me dioscuan ti ta ti vos y cuali ta ti vos seráne ce sario de fi nir los proce sos en tor no a los cua les se ha brá de fun da men tar la per cep ción de la in for ma ción para el fun cio na mien to del sis te ma, para apoyarse en ellos al codificar y representar tanto la descripción del documento como sus contenidos temáticos, así como derivar aquellos elementos cualitativos a incluirse en el deber ser de la normatividad en catalogación.

A pesar de los indiscutibles avan ces que re pre sen tan las nor mas como instrumentos para la organización de catálogos, únicamen te ahon dan en un pro ble ma ya co no ci do, sin de ter mi nar posibles soluciones a cuestiones derivadas de la identificación de un documento durante la exploración previa, es decir, aquella durante la cual el público tiene necesidad de definir los elementos necesarios para orientar el rastreo de información.

Lanormatividad en el ám bi to de la ca ta lo ga ción im plica la definición de un deber ser ineludiblementevinculadoa la determinación de obje ti vos a per se guir por la ca ta lo ga ción, re la ti vos a la cons truc ción de prin ci pios nor ma ti vos cuya in ten ción debe atender a con ver tir la ca ta lo ga ción en un me dio para fa ci li tar la dis tribu ción so cial del co no ci mien to y lo grar que el pú bli co esté bien informado.

Enúl timains tan cia la nor ma ti vi dad enca ta logación for ma par te del de ber ser acerca de las fi na li da des y fun cio nes de la pre servación y or ga ni za ción de do cu men tos, es de cir, fun da men ta su actua ción en fi nes y va lo res so cial men te acep ta dos, de és tos se derivan todos aquellos elementos constitutivos de principios normativos encaminados a establecer una normatividad técnica cuya in ten ción sea ser vir de guía para ins tru men tar una or ga ni zación do cu men tal fun cio nal men te di ri gi da a cum pli men tar los fines y valores de la preservación y difusión de documentos.

Por esta razón la definición de elementos teóricos en catalogación debe concebirse como una función evolutiva, que permita englobar en un todo coherente los elementos relevantes para el cum pli mien to de los fi nes asig na dos a un ca tálo go, sin per der de vista su destinatario final, porque es el público el que en ciertas con di cio nes y en al gún mo men to, le ha brá de dar sig ni fi ca do a la fun ciona li dad for mal del al mace na mien to y recu pe ración docur mental. En este sentido la justificación técnica o la subordinación de la catalogación a intereses profesionales resulta irrelevante o en el mejor de los casos completamente secundaria. 
REFERENCIAS BIBLIOGRAFICAS:

BEA GLE, Do nald. "Li bra ries and the "im pli ca te or der": A con tex tual approach to theory" Libri, v. 38, n. 1, (mar zo 1988) p. $26-44$.

BECK, Helmunt. "The catalogue and the theory, or, Is Order almost a philosophy? On Jean M. Perrault's Lectures The idea of order in bibliography". Int. Classif., v. 14, n. 2, (1987), p. 101-103.

BIBLO, Herbert. “Libra rians and the tra de unionism: A prologue”. Library Trends, v. 25, n. 2, (oc tu ber 1976) p. 423 - 433.

BROOKS, He len. "In for ma tion re trie val and ex pert sys tems- ap proa ches and methods of de volepment" IntelligentInformation retrieval: INFORMATICS, 7. London: ASLIB, 1983, p. 65-78,

CUTTER, Charles A. Rules for a dictionary catalog. 4a. ed. Washington: Goverment Printing Office, 1904. 173 p.

DAVIES (1984), Roy y Brian James. "Towards an expert system for cataloguing: some experimets based on AACR2". Program, v. 18, n. 4, (oct. 1984) p. 283-297.

DA VIES (1986), Roy. "Ex pert sys tems and ca ta lo guing: new wine in old bo ttles?" EnExpert systemsinlibra ries/edi ted by Forbes Gibb. London: Taylor Graham, 1986. p. 67-82.

DA VIES (1987), Roy. “Outli nes of the emer ging pa ra digm in ca ta lo guing”. Information Processing \& Management. v. 23, n. 2 (1987) p. 89-98.

FREEMAN, Maurice J. “The au to mation of ca taloguing-1976.” LibraryTrends, v. 25, n. 3, (ja nuary 1977) p. $703-721$.

GORMAN, Michael. “Chan ges in ca ta lo guing co des: Ru les for entry and hea ding”. Library Trends, v. 25, n. 3, (january 1977) p. 587-601.

HARVARD-Williams, Peter. “International standars” Library Trends, v. 31, n. 1 (summer 1982) p. 173-185

HEN DER SON, Kath ry Luther. "Trea ted with a de gree of uni for mity and common sen ce: Des crip tive cataloguing in the United States, 1876-1975". Library Trends, v. 25, n. 1, (july 1976) p. 227-271.

HICKEY, Do ralyn. “The search for uni for mity in ca ta lo guing: cen tralization and stan dar dization". Library Trends, v. 25, n. 3 (january 1977) p. 565-586.

HOLLEY, Edward G. “Librarians, 1876-1976". Library Trends, v. 25, n. 1, (july 1976) p. 177-207.

INGWERSEN, P. "Search procedures in the library analized from the cognitive point of view". Journal of Documentation, v. 38, (1982) p. 165-191.

MIKHAILOV, A.I. "In for ma tion scien ce and an in for med so ciety: A rus sian in for ma tion scien tist looks at the role of information in society". ASIS Bulletin, v. 10, n. 1 (Octubre 1983) p. 14-17.

SCARPIT, Robert. Teoría general de la información y la comunicación. 2a ed. Barcelona: Icaria, 1981.318 p.

SHAW, Malcom, Bill Dent, David Evans, David Smith. Using AACR2: A diagrammatic Approach. USA: Oryx Press, 1981. viii, 199 p.

SCHUTZ, Al fred.Estudios sobreteoría social. Bue nos Aires: Amorrortu, 1974. Elciu da da no bien in for ma do. En sa yo sobre la distribución social del conocimiento. p. 120-132.

STEVENSON, Gordon. Ru dolph Focke and the theory of the clas sified ca ta log. Illi nois: Uni ver sity of Illi nois, $1980.30 \mathrm{p}$. (Occasional papers, 145 (july 1980))

TA NNEHILL, RO BERT S. y Char les W Hus bands. "Stan dars and bi bli o gra phic data re pre sen tation". Library Trends, v. 31, n. 2, (fall 1982) p. 283-313. 
VICKERY, B. C. "Bibliographic description, arrangement, and retrie val”. JournalofDocumentation. v. 24, n. 1. (mar zo 1968). Reproducido en: introduction to information sciencel compiled and edited by Tefko Saracevic. New York: Bowker, 1970. p. 428-435.

ZWEIZING, Douglas y Eleanor Jo Roger. Output measures for public libraries: a manual of standardized procedures. Chicago: ALA, 1982. 100 p. 